\title{
The Application of the Human Model in the Thermal Comfort Assessment of Fighter Plane's Cockpit
}

\author{
Haifeng Shen and Xiugan Yuan \\ School of Aeronautic Science and Engineering, Beihang University, \\ 100191, Beijing, P.R. China \\ shenhaifeng@139.com
}

\begin{abstract}
Thermal comfort is an important content concerned by aircraft designing and using department. Computational Fluid Dynamics (CFD) is a main numerical simulation method used in this field. A human model of a pilot is built according to its geometrical and thermal features in order to simulate the air flow and heat transfer in a fighter plane's cockpit. The velocity and temperature fields are obtained by using a numerical simulation flat involving the human model above. Meanwhile, an experiment was carried out to prove the effectiveness of numerical simulation. The comparison between calculation results and experiment results shows that the calculation error of velocity and temperature is $15 \%$ and $6 \%$ respectively, which proves that the human model can be used for numerical simulation and satisfy the requirement of simulation precision. Furthermore, some thermal comfort assessment criteria such as the mean skin temperature and the core temperature of the body are obtained by putting calculation result above into the Human Thermal Regulation System (HTRS) model.
\end{abstract}

Keywords: cockpit; CFD; human model; thermal comfort; assessment.

\section{Introduction}

The thermal comfort for a pilot in cockpit is an issue that the plane design and using departments attach great importance to. Velocity and temperature fields formed by the air flow and heat transfer inside a cockpit are the working conditions for a pilot, directly influencing the cockpit's thermal comfort, which then will cause impacts on the pilot's working efficiency [1-2]. For this reason, first we should study the velocity and temperature distribution of airflow inside the cockpit.

Currently, numerical simulation based on CFD is a fairly popular method of solving the air flow and heat transfer in confined space such as rooms, cabins, and so on. Meanwhile, the human model is a key factor in impacting the precision and rationality of a simulation especially when the issue of comfort is involved [3-10].

A human model for a numerical simulation involves both geometrical and thermal features. As the development of 3D modeling technology, the geometrical features of a human model can be generated by using 3D modeling software. However, the thermal features of a human model are relatively difficult to be described for the 
complexity of the heat transfer between human body and external environment. Another difficult point is how to evaluate the thermal comfort after the environment around body is known according to the numerical simulation.

To solve the above-mentioned problems, a human model of a pilot is built for an air velocity and temperature numerical simulation. An experiment was carried out to prove the effectiveness of numerical simulation. Afterwards, an assessment is made by combining the simulation above and the HTRS simulation.

\section{Calculation Models and Methods}

\subsection{Governing Equations}

The basic conservation equations were solved by employing the finite volume and SIMPLEC algorithm. The realizable $k-\varepsilon$ turbulence model was used. The simulation results presented in the paper are steady-state results. The air was assumed to be incompressible and its physical properties were assumed constant. The residuals of continuity, momentum, turbulence kinetic energy and its dissipation rate had to reach $5 \times 10^{-5}$ magnitude for convergence, while the residual of energy had to reach $1 \times 10^{-8}$ in magnitude. The conservation equations were formulated as follows:

$$
\begin{gathered}
\frac{\partial \rho}{\partial t}+\frac{\partial}{\partial x_{j}}\left(\rho u_{j}\right)=0 \\
\frac{\partial}{\partial t}\left(\rho \overline{u_{i}}\right)+\frac{\partial}{\partial x_{j}}\left(\rho \overline{u_{i}} \cdot \overline{u_{j}}\right)=-\frac{\partial p}{\partial x_{i}}+\frac{\partial}{\partial x_{j}}\left[\mu\left(\frac{\partial \overline{u_{i}}}{\partial x_{j}}+\frac{\partial \overline{u_{j}}}{\partial x_{i}}-\frac{2}{3} \delta_{i j} \frac{\partial \overline{u_{k}}}{\partial x_{k}}\right)-\rho \overline{u_{i}^{\prime} u_{j}^{\prime}}\right] \\
\frac{\partial}{\partial t}(\rho \bar{T})+\frac{\partial}{\partial x_{j}}\left(\rho \overline{u_{j}} \cdot \bar{T}\right)=\frac{\partial}{\partial x_{j}}\left[\frac{\mu}{\operatorname{Pr}} \frac{\partial \bar{T}}{\partial x_{j}}-\rho \overline{u_{j}^{\prime} T^{\prime}}\right]+S_{T} \\
\frac{\partial\left(\rho u_{j} k\right)}{\partial x_{j}}=\frac{\partial}{\partial x_{j}}\left[\left(\mu+\frac{\mu_{t}}{\sigma_{k}}\right) \frac{\partial k}{\partial x_{j}}\right]+G_{k}+G_{b}-\rho \varepsilon-Y_{M} \\
\frac{\partial\left(\rho u_{j} \varepsilon\right)}{\partial x_{j}}=\frac{\partial}{\partial x_{j}}\left[\left(\mu+\frac{\mu_{t}}{\sigma_{\varepsilon}}\right) \frac{\partial \varepsilon}{\partial x_{j}}\right]+c_{1} \rho S \varepsilon-c_{2} \rho \frac{\varepsilon^{2}}{k+\sqrt{v \varepsilon}}+c_{1 \varepsilon} c_{3 \varepsilon} \frac{\varepsilon}{k} G_{b}
\end{gathered}
$$

\subsection{Geometrical Features of the Human Model}

When calculating the velocity and temperature fields by CFD method, grid generation is the first step. To generate high quality grid, a good geometrical model is an 
important precondition. From the angle of geometrical modeling, the human model is the most difficult part of the whole cockpit geometry. First, the geometry of human body is so complicated that it is hard to be modeled accurately. In fact, the more complicated the geometrical model is, the more difficult it is to generate the grid. Even if the grid can be generated, some deformed cells of grid will probably be produced. Consequently, the convergence and accuracy of numerical simulation will be affected remarkably. On the other hand, if the geometry of human body is oversimplified and some key features are lost, the simulation will not describe the real picture of velocity and temperature fields.

Therefore, a reasonable geometry of human model was built according to the standard human body data in Military Criterion [11-12] in this research. Relatively, the sizes and surface features of several main body parts were described precisely, while other unnecessary details were ignored. Moreover, the actual working gestures were taken into consideration in designing. As a result, the human model's geometrical size, shape and gesture were comparatively closer to the real situation. A fighter plane cockpit's configuration with human body is indicated in Fig.1.

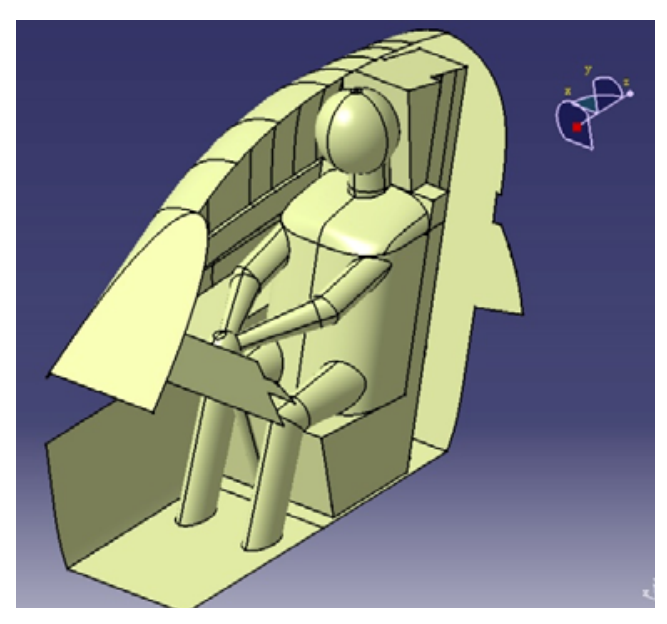

Fig. 1. Geometry of cockpit with human body

Based on the geometrical model above, unstructured multi-block grid was used to solve the problem. The grid contains 1123265 cells.

Supply air travels from a main pipe lying on the left floor, and then is divided into five branches on its way including back hatch pipe, windshield pipe, feet pipe, left nozzle and right nozzle. The outlet is on the right side of the back bulkhead. 


\subsection{Thermal Features of the Human Model}

Heat transfer boundary conditions are critical foundations in CFD. A reasonable heat transfer boundary condition should reflect the true thermal features of the boundary and the true heat transfer process between the boundary and the environment. For its physiological characteristics, the thermal features of a human body are too complicated to be described by a simple boundary condition. In past researches, a human body is generally dealt with as an isothermal wall or a uniform heat flux wall. However, each part of the body differs much in their respective physiological characteristic and heat transfer process, so the simplified way above is unreasonable.

In this research, a human body was divided into four parts: head, trunk, arms and legs. The generated heat of each part is showed in Table 1. And then each part can be regarded as a uniform heat flux wall.

Table 1. Generated heat of each part of the body

\begin{tabular}{ccccc}
\hline Body Part & Head & Trunk & Arms & Legs \\
\hline Ratio(\%) & 18.4 & 66 & 7.3 & 8.3 \\
Total Power & \multicolumn{4}{c}{$150 \mathrm{~W}$} \\
\hline
\end{tabular}

\section{Experiment}

\subsection{Measurement Planes and Points}

Measurement planes chosen for the experiment on measuring velocity and temperature fields are shown as Fig. 2 in which plane 1 is the symmetry plane of the cockpit $(\mathrm{x}=0)$ and plane 2 is in front of human body and the vertical distance between plane 2 and body is about 50 80mm, in addition, a region near cloth surface was settled for temperature field measurement. Measurement points on each plane or region are indicated in Fig.3.

\subsection{Heat Load Simulation and Measurement Equipment}

Aerodynamic heat load and generated heat load of human body were simulated in the experiment. Aerodynamic heat load was simulated by using radiation heating chamber to heat outside surface of cockpit and the temperature of outside surface was controlled according to the simulation flying condition. Generated heat load was simulated by using a dummy wrapped in heating tape and heat power of each part is shown in Table 1.

ATM2400 36-channel airflow and temperature instrument produced by DegreeC company in U.S.A was used to measure velocity field and temperature field in the cockpit. 


\section{Comparison between Numerical and Experimental Data}

Under the condition of air demand $G_{0}=300 \mathrm{~kg} / \mathrm{h}$ and both nozzles closed. The temperature contours of measurement plane 1 and plane 2 are presented as Fig.4, Fig.5 respectively. The comparisons of temperature fields between numerical and experimental results are presented as Fig.6.

Under this condition, the calculation error of velocity field of plane1 and plane 2 compared with experimental results is $13.48 \%$ and $18.46 \%$ respectively, and the calculation error of temperature field of plane1, plane 2 and the region near cloth surface is $5.24 \%, 5.70 \%$ and $8.63 \%$. This shows that human model built above can satisfy the requirement of numerical simulation, which means that the geometrical and thermal features settled above are reasonable.

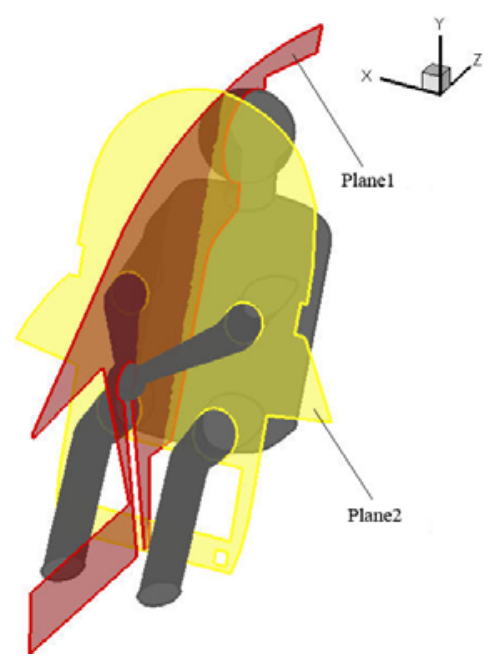

Fig. 2. Measurement planes

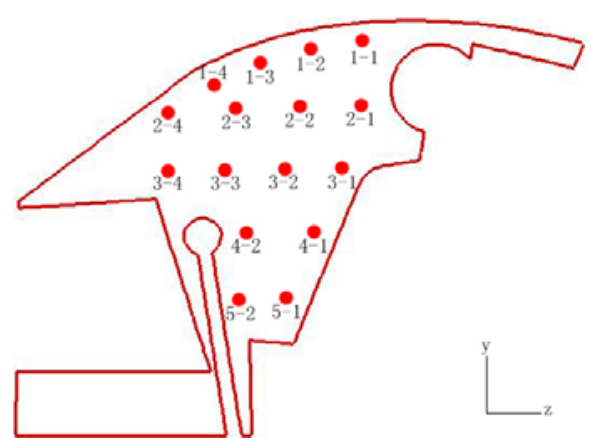

(a) Plane 1 


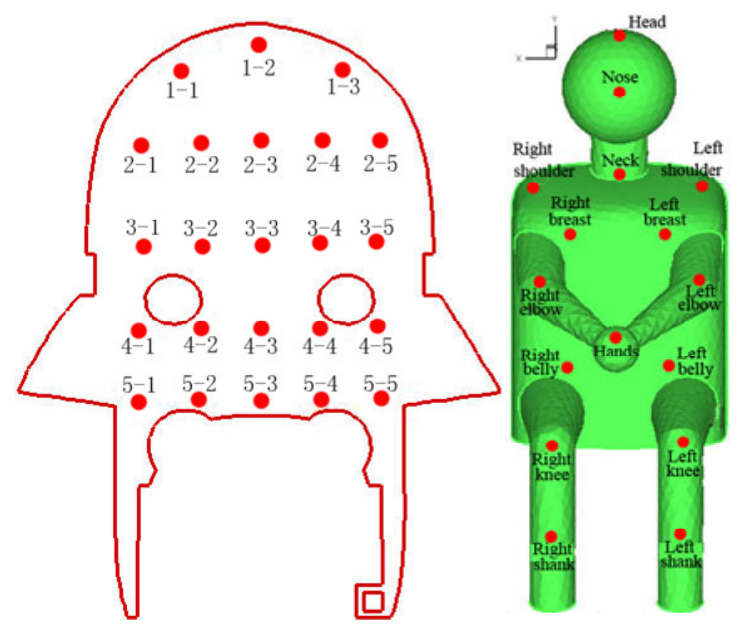

Fig. 3. Measurement points

\section{Thermal Comfort Assessment}

\subsection{Thermal Comfort Assessment Criteria}

After the velocity and temperature fields in the cockpit are calculated by the above method, the calculation results can be used to assess thermal comfort of the cockpit by referring to the relative standards. There are two assessment methods: one is the mean temperature near the body $\left(\bar{T}_{d b}\right)$ and the other is a physiological method including the mean skin temperature $\left(\bar{T}_{\text {skin }}\right)$ and the core temperature $\left(T_{\text {core }}\right)$. The thermal comfort standards of these three assessment criteria are indicated in Table 2 [13]. $\bar{T}_{d b}$ can be calculated from numerical simulation results of the temperature field directly, but $\bar{T}_{\text {skin }}$ and $T_{\text {core }}$ must be obtained by putting calculation results above into the HTRS model.

Table 2. Thermal comfort assessment standards of the cockpit

\begin{tabular}{cccc}
\hline \multirow{2}{*}{ Assessment Criteria } & \multicolumn{3}{c}{ Temperature Range } \\
\cline { 2 - 4 } & Feeling comfort & Ensuring efficiency & Slight stress \\
\hline $\bar{T}_{d b},{ }^{\circ} \mathrm{C}$ & $16.0-26.0$ & $27.0-32.0$ & $33.0-35.0$ \\
$T_{\text {core }},{ }^{\circ} \mathrm{C}$ & $37.0 \pm 0.2$ & $37.0 \pm 0.4$ & $>37.6$ or $<36.4$ \\
$\bar{T}_{\text {skin }},{ }^{\circ} \mathrm{C}$ & $33.0 \pm 1.5$ & $33.0 \pm 2.6$ & $33.0 \pm 4.0$ \\
\hline
\end{tabular}




\subsection{Human Thermal Regulation System Model [14]}

The actual HTRS is very complicated and can be regarded as a feedback system. The whole system has two subsystems, which are control system and controlled system. Control system means nervous system participating in temperature regulation including regulation center and thermorecceptors. Controlled system is made up of effectors. In HTRS, the controlled variables are $\bar{T}_{\text {skin }}$ and $T_{\text {core }}$. Regulation center collects and integrates all signals received by thermorecceptors, and then drives relevant effectors to generate physiologic reaction of temperature regulation. Therefore, if environment temperature figures can be obtained, $\bar{T}_{\text {skin }}$ and $T_{\text {core }}$ will be calculated by using HTRS model.

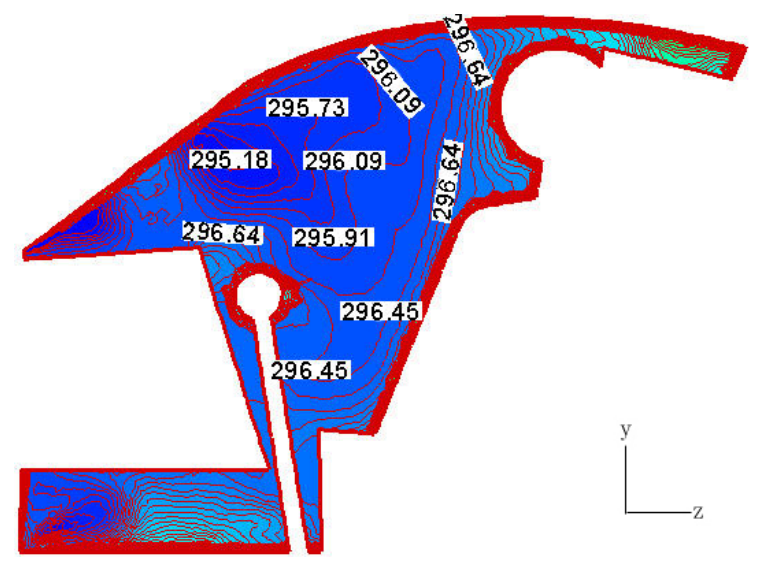

(a) Calculation result

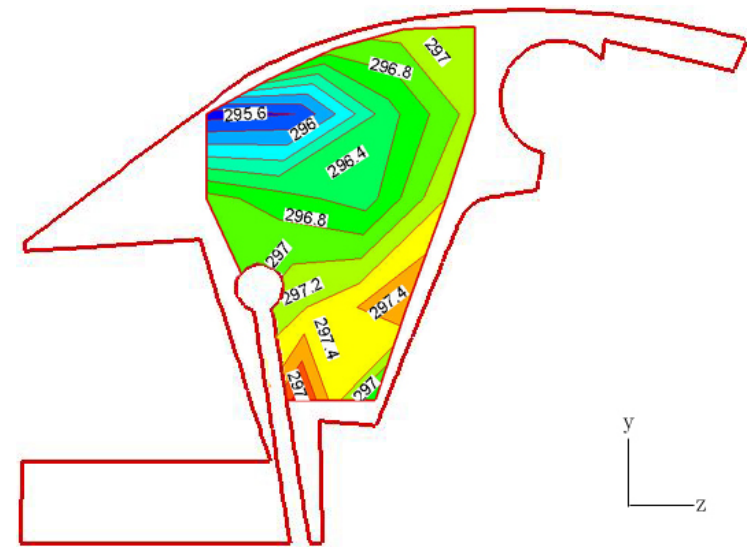

(b) Experiment result

Fig. 4. Temperature contour of measurement plane $1, G_{0}=300 \mathrm{~kg} / \mathrm{h}$ and both nozzles closed 


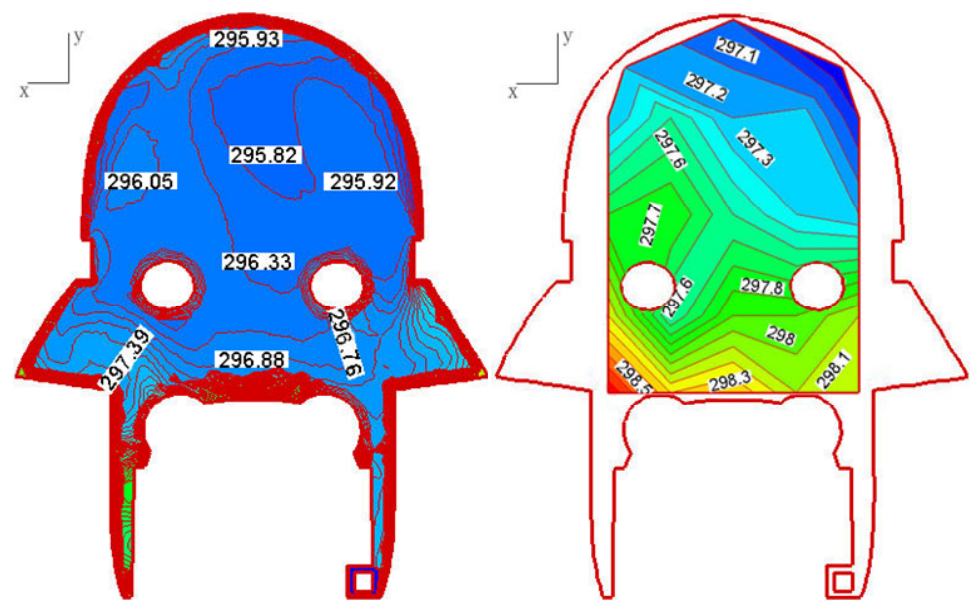

(a) Calculation result

(b) Experiment result

Fig. 5. Temperature contour of measurement plane $2, G_{0}=300 \mathrm{~kg} / \mathrm{h}$ and both nozzles closed

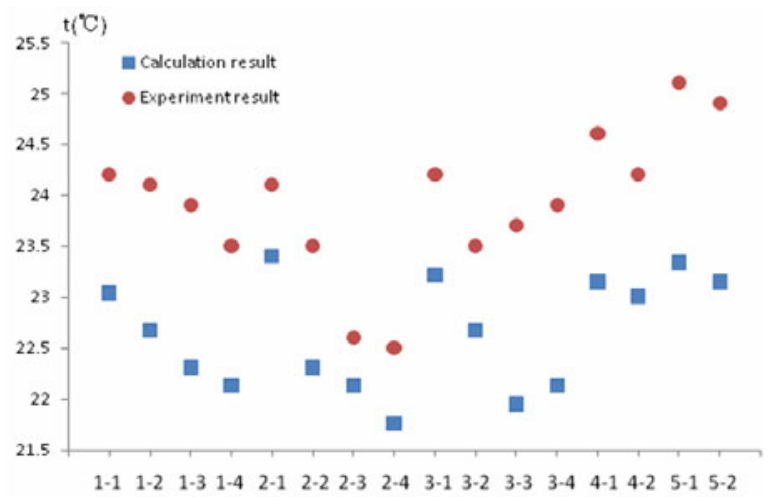

(a) Plane 1

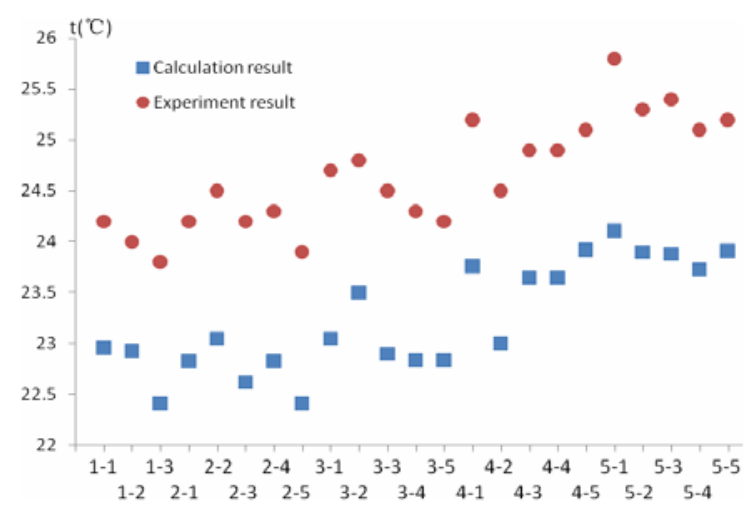

(b) Plane2 


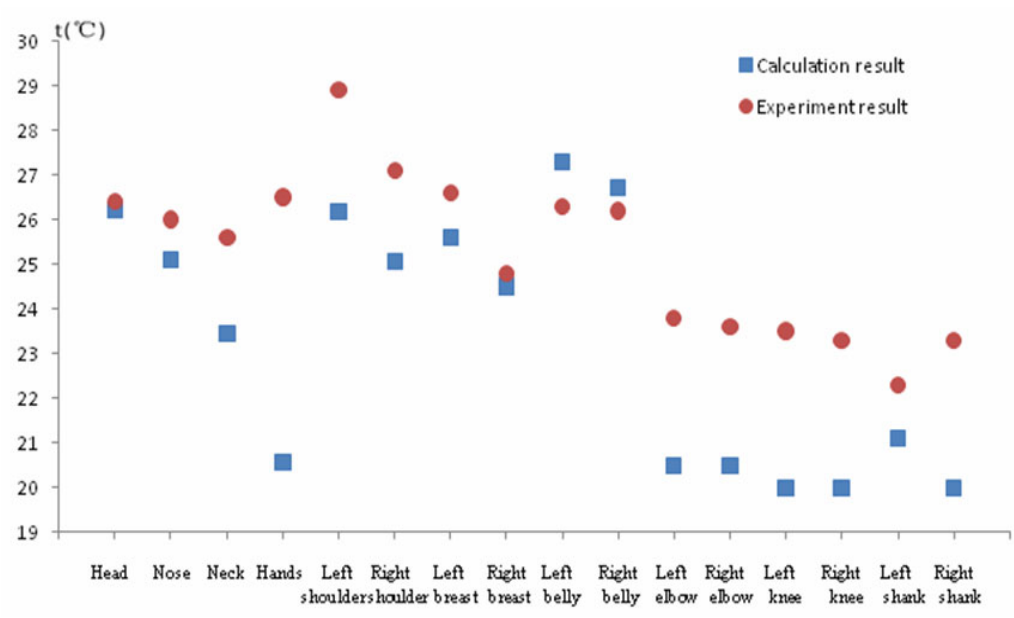

(c) Near cloth surface

Fig. 6. Comparison between calculation and experiment results of temperature field, $G_{0}=300 \mathrm{~kg} / \mathrm{h}$ and both nozzles closed

\subsection{Thermal Comfort Assessment Analysis}

According to the velocity and temperature fields obtained above and HTRS model, $\bar{T}_{d b}, \bar{T}_{s k i n}$ and $T_{\text {core }}$ can be calculated. The results are indicated in Table 3. Compared with Table 3, we know that under the condition of air demand $G_{0}=300 \mathrm{~kg} / \mathrm{h}$ and both nozzles closed, the three assessment criteria above are all in the range of feeling comfort, which means that the temperature environment in the cockpit can satisfy the thermal comfort requirement.

Table 3. Calculation results of thermal comfort assessment criteria

\begin{tabular}{ccc}
\hline $\bar{T}_{d b},{ }^{\circ} \mathrm{C}$ & $T_{\text {core }},{ }^{\circ} \mathrm{C}$ & $\bar{T}_{\text {skin }},{ }^{\circ} \mathrm{C}$ \\
\hline 23.3 & 37.03 & 33.68 \\
\hline
\end{tabular}

\section{Conclusions}

The following conclusions can be obtained by this investigation:

(1) The human model is a key factor of the numerical simulation about air flow and heat transfer in confined space with human body inside, and a human model with reasonable geometrical and thermal features can improve the simulation accuracy;

(2) The thermal comfort assessment criteria can be calculated by combining the numerical simulation of air flow and heat transfer and HTRS model. This means if physiologic features are taken into consideration, an integrated human model can be built and a complete method of thermal comfort assessment can be established accordingly. 


\section{References}

1. Chen, X., Yuan, X.: General human-machine-environment system. Beihang University Press, Beijing (2000) (in Chinese)

2. Shou, R., He, H.: Aircraft's environment control. Beihang University Press, Beijing (2004) (in Chinese)

3. DeJager, A.W., Lytle, D.B.: Commercial airplane airdistribution system development through the use of computational fluid dynamics. In: AIAA-1992-0987, pp. 1-9 (1992)

4. Singh, A., Hosni, M.H., Hortsman, R.H.: Numerical simulation of airflow in an aircraft cabin section. ASHRAE Transactions 108(1), 1005-1013 (2002)

5. Gunther, G., Bosbach, J., Pennecot, J., et al.: Experimental and numerical simulations of idealized aircraft cabin flows. Aerospace Science and Technology (10), 563-573 (2006)

6. Zhang, T.F., Chen, Q.Y.: Novel air distribution systems for commercial aircraft cabins. Building and Environment (42), 1675-1684 (2007)

7. Yuan, X.: Study on Mathematic Model of Human-Cockpit's Environment Temperature. Selected Papers of The Key Research Topic of Human-Machine-Environment Engineering, pp. 17-23 (1987) (in Chinese)

8. Yang, C.: The numerical simulation on the thermal behavior of aircraft cabin. Acta Aeronauticaet Astronautica Sinica 16(1), 64-68 (1995) (in Chinese)

9. Lin, G.: Numerical fluid flow and heat transferwithin an air-conditioning cockpit. School of Aeronautic Science and Engineering. Beihang University, Beijing (1998) (in Chinese)

10. Xiong, X., Liu, W., Ang, H., et al.: Flow fields and thermal comfort in training-plane's airconditioned cockpit. J. Applied Sciences 25(6), 639-644 (2007)

11. Technology and Industry for National Defense. GJB20-84, Standard series on individual protecting and lifesaving equipment of pilot. Military Criterion Press of Technology and Industry for National Defense, Beijing

12. Technology and Industry for National Defense. GJB36-85, The design and use requests of pilot's body model. Military Criterion Press of Technology and Industry for National Defense, Beijing

13. Technology and Industry for National Defense. GJB1129-91, Methodology and physiological requirements for temperature assessments of cockpit in military aircraft. Military Criterion Press of Technology and Industry for National Defense, Beijing

14. Qiu, Y.: Numerical and experimental study on liquid cooling garment used for extravehicular activity - human thermal regulation system. School of Aeronautic Science and Engineering, Beihang University, Beijing (2002) (in Chinese) 